\title{
Prognostic significance of the FLT3 ITD mutation in patients with normal-karyotype acute myeloid leukemia in relapse
}

\author{
Sang Hyuk Park ${ }^{1}$, Hyun-Sook Chi ${ }^{1}$, Sook-Kyung Min ${ }^{1}$, Young-Uk Cho ${ }^{2}$, Seongsoo Jang ${ }^{1}$, Chan-Jeoung \\ Park ${ }^{1}$, Jung-Hee Lee ${ }^{3}$, Je-Hwan Lee ${ }^{3}$, Kyoo-Hyung Lee ${ }^{3}$, Ho-Joon $\operatorname{Im}^{4}$, Jong-Jin Seo ${ }^{4}$ \\ ${ }^{1}$ Department of Laboratory Medicine, University of Ulsan College of Medicine and Asan Medical Center, ${ }^{2}$ Department of Laboratory \\ Medicine, Eulji University School of Medicine, Eulji General Hospital, Departments of ${ }^{3}$ Internal Medicine and ${ }^{4}$ Pediatrics, University \\ of Ulsan College of Medicine and Asan Medical Center, Seoul, Korea
}

p-ISSN 1738-7949 / e-ISSN 2092-9129

DOI: $10.5045 / \mathrm{kjh} .2011 .46 .2 .88$

Korean J Hematol 2011;46:88-95.

Received on March 10, 2011

Revised on April 11, 2011

Accepted on May 17, 2011

\author{
Correspondence to \\ Hyun-Sook Chi, M.D., Ph.D. \\ Department of Laboratory Medicine, Asan \\ Medical Center and University of Ulsan \\ College of Medicine, 86, Asanbyungwon- \\ gil, Songpa-gu, Seoul 138-736, Korea \\ Tel: $+82-2-3010-4502$ \\ Fax: +82-2-478-0884 \\ E-mail: hschi@amc.seoul.kr \\ (C) 2011 Korean Society of Hematology
}

\section{Background}

Fms-like tyrosine kinase 3 internal tandem duplication (FLT3 ITD) mutation is related to poor prognosis in normal-karyotype acute myeloid leukemia (AML). However, the prognostic significance of the mutation at relapse has not been adequately investigated. We investigated the prognostic significance of the FLT3 ITD mutation at relapse in normal-karyotype AML patients.

\section{Methods}

We analyzed 69 normal-karyotype AML patients, in whom paired bone marrow samples taken at initial diagnosis and subsequent relapse were analyzed for the FLT3 ITD mutation at the Asan Medical Center between 1995 and 2009.

\section{Results}

Forty patients showed a persistent wild-type genotype, 11 showed the FLT3 ITD mutation at diagnosis and relapse, and 9 lost and another 9 acquired the mutation at relapse. The mutation status at relapse affected the overall survival (OS), with the mutation group showing shorter OS and survival after relapse than the wild-type group did $(P<0.001$ and $P<0.001$, respectively), despite having received more frequent stem cell transplantation after relapse than the wild-type group did. However, no difference was detected in the OS and survival after relapse with regard to the mutation status at diagnosis.

\section{Conclusion}

The patients with FLT3 ITD mutation at relapse showed poorer prognoses than those without the mutation. However, mutation status at diagnosis did not affect the outcome. These results suggest that, in normal-karyotype AML patients with relapse, the prognostic significance of FLT3 ITD mutation at relapse is greater than that of the mutation status at diagnosis.

Key Words AML, Prognosis, FLT3 ITD, Relapse, Normal karyotype

\section{INTRODUCTION}

The Fms-like tyrosine kinase 3 (FLT3) gene encodes a class III receptor tyrosine kinase that plays important roles in cellular proliferation and differentiation [1-8]. To date, 2 types of FLT3 mutations have been found to induce autophosphorylation through ligand-independent FLT3 dimerization, leading to uncontrolled hematologic progenitor cell proliferation and malignancy. One such mutation is an internal tandem duplication (ITD) of the region between exon
14 and 15 encoding the juxtamembrane domain; the other, in exon 20, is a point mutation in aspartic acid residue 835 (D835) within the activation loop of the second tyrosine kinase domain $[9,10]$. The FLT3 ITD mutation is found in approximately $30 \%$ of newly diagnosed acute myeloid leukemia (AML) patients, and an association of the mutation with poor prognostic indicators, such as leukocytosis, higher marrow blast counts, shorter overall survival (OS) and event-free survival (EFS), and higher relapse rates, has been firmly established in those with newly diagnosed AML, especially in the intermediate cytogenetic risk group [11-16].

This is an Open Access article distributed under the terms of the Creative Commons Attribution Non-Commercial License (http://creativecommons.org/licenses/by-nc/3.0) which permits unrestricted non-commercial use, distribution, and reproduction in any medium, provided the original work is properly cited. 
The FLT3 D835 mutation occurs less frequently than the FLT3 ITD mutation does, and the prognostic significance of this mutation in newly diagnosed AML patients is less clear, with previous studies reporting conflicting outcomes [17-19]. In addition to the FLT3 ITD mutation, mutations in the nucleophosmin1 (NPM1) gene are the most frequent genetic alterations, being found in approximately $50 \%$ of patients with normal-karyotype AML, and known to be associated with better response to induction therapy and a more favorable outcome in the absence of FLT3 ITD mutations [20].

Thus far, the prognostic impact of the FLT3 ITD mutation at relapse has not been adequately evaluated in patients with normal-karyotype AML. Therefore, we analyzed FLT3 ITD mutation status at diagnosis and relapse in patients with normal-karyotype AML relapse, and investigated the prognostic significance of FLT3 ITD mutation at relapse.

\section{MATERIALS AND METHODS}

1. Selection of patients and assessment of clinical variables

Paired bone marrow samples collected at diagnosis and relapse from 69 patients newly diagnosed with normal-karyotype AML between 1995 and 2009 at Asan Medical Center were analyzed for FLT3 ITD mutation status. Data for clinical variables, including $\mathrm{CBC}$, blast counts in peripheral blood $(\mathrm{PB})$ and bone marrow (BM), karyotype at initial diagnosis and relapse, and implementation of stem cell transplantation (SCT) were obtained from all patients. The dates of initial diagnosis, first complete remission (CR), first relapse, SCT performance, and death were established by performing a retrospective review of the patients' medical records. CR was defined as the presence of less than $5 \%$ blasts with more than $20 \%$ cellularity in a standardized BM aspirate after the first or second course of induction chemotherapy. Relapse was defined as more than 5\% leukemic blasts in $\mathrm{BM}$ aspirates in patients who had previously achieved CR state. OS was defined as the time from diagnosis to death or last follow-up, and survival after relapse was defined as the time from first relapse to death or last follow-up.

\section{Cytogenetic analysis}

Cytogenetic analyses were performed on at least 20 metaphases using paired diagnosis and relapse BM samples. According to the classification system proposed by the US Southwest Oncology Group (SWOG), cytogenetic abnormalities at relapse were grouped into 4 categories [21]: (1) goodprognosis: $\operatorname{inv}(16) / \mathrm{t}(16 ; 16) / \operatorname{del}(16 \mathrm{q})$ or $\mathrm{t}(8 ; 21)$ lacking $\operatorname{del}(9 \mathrm{q})$ and complex karyotypes; (2) intermediate-prognosis: normal karyotype, $+8,+6$, Y, and del(12p); (3) poor-prognosis: 5/del (5q), 7/del(7q), abn(3q), t(6;9) and complex karyotypes; (4) unknown-prognosis: all the other above-mentioned abnormalities.

\section{Patient treatment}

All patients received standard induction chemotherapy with cytarabine and daunorubicin. This regimen included continuous intravenous infusion of $200 \mathrm{mg} / \mathrm{m}^{2} /$ day $(100 \mathrm{mg} /$ $\mathrm{m}^{2} /$ day for patients aged $>60$ years) of cytarabine on days $1-7$ and $45 \mathrm{mg} / \mathrm{m}^{2} /$ day of daunorubicin on days $1-3$. Patients who failed to achieve $\mathrm{CR}$, but attained partial remission $(\mathrm{PR})$ received a second identical cycle of induction chemotherapy. At relapse, the patients received the same regimen of induction chemotherapy used following the initial diagnosis. Depending on patient age and availability of a suitable donor, the patients received autologous or allogeneic SCT on the first CR status or later. Patients with FLT3 ITD mutation at initial diagnosis were preferentially invited to receive autologous or allogeneic SCT at the first CR status. However, there was no difference in the induction chemotherapy regimen according to FLT3 ITD mutation status at initial diagnosis or relapse.

\section{Detection of FLT3 ITD and NPM1 mutations}

FLT3 ITD and NPM1 mutations were identified using PCR-capillary electrophoresis methods. Genomic DNA was extracted from BM mononuclear cells using the Qiagen DNA purification kit (Qiagen, Hilden, Germany) and stored at $-20^{\circ} \mathrm{C}$. Primer sets were designed to detect the FLT3 ITD and NPM1 4 base pair insertion mutations. Additionally, a specific set of $H B G$ primers was used to ascertain DNA integrity and validate the precision of PCR amplification. The sequences of the primers specific to FLT3, NPM1, and $H B G$ were as follows. FLT3 primers: forward, 5'-FAM-AGCA ATT TAG GTA TGA AAG CCA GCTA-3'; reverse, 5'-CTT TCA GCA TTT TGA CGG CAA CC-3'. NPM1 primers: forward, 5'-GTT TCT TTT TTT TTT TTT CCA GGC TAT TCA AG-3', reverse, 5'-HEX-CAC GGT AGG GAA AGT TCT CAC TCT GC-3'. HBG primers: forward, 5'-HEX-CCA GAA GAG CCA AGG ACA GGT ACG-3', reverse, 5'-AGA TCC CCA AAG GAC TCA AAG AAC C-3'. The $20 \mu \mathrm{L}$ multiplex PCR reaction solution consisted of $200 \mathrm{ng}$ of genomic DNA, $20 \mathrm{pmol}$ of each primer, $25 \mathrm{mmol} / \mathrm{L} \mathrm{MgCl}_{2}, 2.5 \mathrm{mmol} / \mathrm{L}$ dNTP, $2 \mu \mathrm{L}$ of 10× PCR buffer, $0.2 \mu \mathrm{L}$ of Qiagen HotTaq DNA polymerase and water. The PCR conditions were as follows: the mixture was heated to $95^{\circ} \mathrm{C}$ for $15 \mathrm{~min}$ for initial denaturation, and then 35 cycles of $95^{\circ} \mathrm{C}$ for $20 \mathrm{sec}$, $60^{\circ} \mathrm{C}$ for $40 \mathrm{sec}$, and $72^{\circ} \mathrm{C}$ for $40 \mathrm{sec}$ were performed, followed by a final extension phase of $72^{\circ} \mathrm{C}$ for $30 \mathrm{~min}$. The PCR products were then diluted 1:30 and analyzed by capillary electrophoresis using ABI 310 genetic analyzer (Applied Biosystems Inc., Foster city, CA, USA) and GeneScan Analysis software (version 1.2, Applied Biosystems Inc., Foster City, CA, USA). The data is represented as a ratio of mutant cells:wild-type cells. The lowest detection limit of this protocol was $5 \%$, which had been determined by previous serial dilution experiments [22]. For the specimens, in which NPM1 mutation was detected, additional direct sequencing was performed to confirm the mutation subtype.

5. Analysis of clinical variables related to FLT3 ITD paired mutation status at diagnosis and relapse

An analysis of clinical variables related to FLT3 ITD muta- 
tion status at diagnosis and relapse was performed. Results were grouped into 4 categories: (1) persistent wild type: NN (negative at diagnosis and relapse); (2) acquired mutation: NP (negative at diagnosis and positive at relapse); (3) loss of mutation: $\mathrm{PN}$ (positive at diagnosis and negative at relapse); and (4) persistent mutation: PP (positive at diagnosis and relapse). The clinical variables described above were compared individually between $\mathrm{NN}$ and $\mathrm{PN}, \mathrm{NN}$ and $\mathrm{NP}, \mathrm{NN}$ and $\mathrm{PP}$, and $\mathrm{PN}$ and $\mathrm{PP}$ groups. Two additional comparisons were made: between patients positive for FLT3 ITD mutation at relapse $(\mathrm{NP}+\mathrm{PP})$ and those negative for FLT3 ITD mutation at relapse $(\mathrm{NN}+\mathrm{PN})$, and between patients positive for FLT3 ITD mutation at diagnosis $(\mathrm{PN}+\mathrm{PP})$ and those negative for FLT3 ITD mutation at diagnosis (NN+NP). Additionally, we compared the prognosis in patients without FLT3 ITD mutation stratifying according to NPM1 mutation status at diagnosis or relapse to identify the prognostic impact of NPM1 mutations. We also compared the prognosis of patients on the basis of FLT3 ITD mutant:wild-type ratio in patients with the mutation at diagnosis or relapse in order to evaluate the effect of the amount of mutation on the prognosis.

6. Multivariate analysis of $O S$ and survival after relapse with respect to variables, including FLT3 ITD mutation status at diagnosis and relapse

Multivariate analysis of OS and survival after relapse with respect to FLT3 ITD mutation status at diagnosis and relapse was performed. For the analysis at diagnosis, WBC counts and blast percentage in $\mathrm{PB}$ and $\mathrm{BM}$, which were found to be significantly different among FLT3 ITD mutation groups in univariate analysis, were used to adjust the relevant outcomes. For the analysis at relapse, duration of CR and SCT performance rates after relapse, which showed significant differences among FLT3 ITD mutation groups in univariate analysis, were used to adjust the dependent measures.

\section{Statistical analysis}

Pearson's chi-squared or Fisher's exact tests (for small numbers) were used to compare categorical variables. MannWhitney $U$ test was used to compare continuous variables. Estimations of OS and survival after relapse were performed using Kaplan-Meier methods and compared using log-rank test. Multivariate analyses of OS and survival after relapse were performed using Cox's proportional hazards model. For all analyses, tests were two-tailed, and $P$-values $\leq 0.05$ were considered statistically significant. All calculations were performed using SPSS 13.0.1 for Windows (SPSS Inc, Chicago, IL, USA).

\section{RESULTS}

\section{Overall patient characteristics}

All patients achieved first CR after a median interval of 32.0 days and relapsed within a median of 7.6 months from the first CR. A second CR was achieved in 26 patients (37.7\%), of whom $8(30.8 \%)$ relapsed again after the second round of induction chemotherapy. Fifty-five patients (79.7\%) died after relapse with a median interval of 3.8 months. The FLT3 ITD mutation was detected in 20 patients (29.0\%) at diagnosis and relapse, with median mutant:wild-type ratio as 0.3 (range, 0.05-4.36) and 0.3 (range, 0.05-9.94) at diagnosis and relapse, respectively. The NPM1 mutation was detected in $19(27.5 \%)$ and $13(18.8 \%)$ patients at diagnosis and relapse, with median mutant:wild-type ratio as 0.55 (range, 0.16-0.86) and 0.55 (range, 0.07-0.75) at diagnosis and relapse, respectively. Among the 19 patients who showed NPM1 mutation at diagnosis, $16(84.2 \%)$ had NPM1 type-A mutation. Among the 13 patients who showed $N P M 1$ mutation at relapse, $10(76.9 \%)$ had NPM1 type-A mutation. The remaining 3 patients had NPM1 type-D mutation at diagnosis and relapse. Cases showing a change of NPM1 mutation type from diagnosis to relapse were not found.

2. Comparison of clinical variables and prognosis between patients with different paired FLT3 ITD mutation status Tables 1 and 2 show comparisons of the clinical variables and prognosis between patients with different FLT3 ITD mutation status at diagnosis and relapse. SCT performance rates at the first CR ranged from $25.0 \%$ to $44.4 \%$, which is variable but this variation was not driven by FLT3 ITD mutation status at diagnosis and relapse. However, SCT performance rates after the first relapse were significantly different. Patients with acquired or persistent FLT3 ITD mutation at relapse more frequently underwent SCT than those with persistent negative status did $(55.6 \%$ or $63.6 \%$ vs. $20.0 \% ; P=0.043,0.009$, respectively). There was no prognostic impact of being in the poor cytogenetic risk group at relapse (OS: 20.6 vs. 22.0 months, $P=0.950$; duration of CR: 7.5 vs. 8.9 months, $P=0.669$; survival after relapse: 10.2 vs. 14.3 months, $P=0.214$ ).

The PP group showed a significantly shorter median duration of CR (6.9 months) than the NN group (10.3 months; $P=0.045)$; the PP group also showed a significantly higher median WBC count $\left(44.1 \times 10^{9} / \mathrm{L}\right.$ vs. $\left.8.8 \times 10^{9} / \mathrm{L} ; P=0.046\right)$ than the NN group, but these variables did not differ between the PP and PN groups. The PN group showed significantly higher median WBC counts $\left(66.1 \times 10^{9} / \mathrm{L}\right.$ vs. $8.8 \times 10^{9} / \mathrm{L}$; $P=0.011)$ and blast percentages in $\mathrm{PB}(85.0 \%$ vs. $38.5 \%$, $P=0.006)$ and in $\mathrm{BM}(87.0 \%$ vs. $60.2 \%, P=0.001)$ than the NN group. The median OS was significantly shorter in the NP (9.2 months; $P=0.002)$ and the PP (12.1 months; $P=0.003$ ) groups than in the NN group (19.5 months). The median survival after relapse was also significantly shorter in the NP (5.3 months; $P=0.017$ ) and the PP (4.9 months; $P=0.024$ ) groups than in the NN group (10.3 months). The median OS and survival after relapse in the PP group was significantly shorter than that in the PN group (45.5 months and 43.5 months; $P=0.025$ and 0.028 , respectively).

3. Comparison of clinical variables and prognosis between groups with different FLT3 ITD mutation status at diagnosis and relapse

Table 3 shows 2 additional comparisons of clinical variables 
Table 1. Comparison of clinical variables between patients with different paired FLT3 ITD mutation status.

\begin{tabular}{|c|c|c|c|c|c|c|c|c|}
\hline \multirow{2}{*}{ Clinical variables } & \multicolumn{4}{|c|}{$\begin{array}{c}\text { Number of patients with FLT3 ITD mutation status } \\
\text { (\% with each status) }\end{array}$} & \multicolumn{4}{|c|}{$P$} \\
\hline & NN & PN & NP & PP & NN vs. PN & NN vs. NP & NN vs. PP & PN vs. PP \\
\hline $\operatorname{Sex}(M: F)$ & $25: 15$ & $5: 4$ & $4: 5$ & $8: 3$ & ns & ns & ns & ns \\
\hline Age, median (range) & $\begin{array}{c}54.0 \\
(6.0-79.0)\end{array}$ & $\begin{array}{c}41.0 \\
(27.0-70.0)\end{array}$ & $\begin{array}{c}59.0 \\
(5.0-73.0)\end{array}$ & $\begin{array}{c}45.0 \\
(23.0-73.0)\end{array}$ & ns & ns & ns & ns \\
\hline \multicolumn{9}{|l|}{ FAB classification } \\
\hline Mo & $2(5.0)$ & 0 & 0 & 0 & & & & \\
\hline M1 & $11(27.5)$ & $5(55.6)$ & $2(22.2)$ & $2(18.2)$ & & & & \\
\hline M2 & 15 (37.5) & $3(33.3)$ & $5(55.6)$ & $3(27.3)$ & & & & \\
\hline M4 & $3(7.5)$ & 0 & $1(11.1)$ & $6(54.5)$ & & & & \\
\hline M5 & $1(2.5)$ & $1(11.1)$ & 0 & 0 & & & & \\
\hline M6 & $4(10.0)$ & 0 & 0 & 0 & & & & \\
\hline M7 & $4(10.0)$ & 0 & $1(11.1)$ & 0 & & & & \\
\hline \multicolumn{9}{|l|}{ Karyotype at relapse } \\
\hline Good & 0 & 0 & 0 & 0 & & & & \\
\hline Intermediate & $29(72.5)$ & 8 (88.9) & $7(77.8)$ & 7 (63.6) & & & & \\
\hline Poor & 5 (12.5) & $1(11.1)$ & 0 & $1(9.1)$ & & & & \\
\hline Unknown & $6(15.0)$ & 0 & $2(22.2)$ & $3(27.3)$ & & & & \\
\hline \multicolumn{9}{|l|}{ Clonal evolution } \\
\hline No & $27(67.5)$ & $8(88.9)$ & $7(77.8)$ & 7 (63.6) & & & & \\
\hline Yes & $13(32.5)$ & $1(11.1)$ & $2(22.2)$ & $4(36.4)$ & & & & \\
\hline \multicolumn{9}{|l|}{ SCT in $1^{\text {st }}$ CR status } \\
\hline No & $30(75.0)$ & $5(55.6)$ & $6(66.7)$ & $7(63.6)$ & ns & ns & ns & ns \\
\hline Yes & $10(25.0)$ & $4(44.4)$ & $3(33.3)$ & $4(36.4)$ & & & & \\
\hline \multicolumn{9}{|l|}{ SCT after $1^{\text {st }}$ relapse } \\
\hline No & $32(80.0)$ & $7(77.8)$ & $4(44.4)$ & $4(36.4)$ & ns & 0.043 & 0.009 & $\mathrm{~ns}$ \\
\hline Yes & $8(20.0)$ & $2(22.2)$ & $5(55.6)$ & $7(63.6)$ & & & & \\
\hline \multicolumn{9}{|l|}{ NPM1 mutation status } \\
\hline Persistent wild-type (NN) & 35 (87.5) & $6(66.7)$ & $6(66.7)$ & $3(27.3)$ & & & & \\
\hline Lost at relapse (PN) & $2(5.0)$ & $2(22.2)$ & 0 & $2(18.2)$ & & & & \\
\hline Persistent mutation (PP) & $3(7.5)$ & $1(11.1)$ & $3(33.3)$ & $6(54.5)$ & & & & \\
\hline \multicolumn{9}{|c|}{ Laboratory findings at diagnosis, median } \\
\hline $\mathrm{Hb}(\mathrm{g} / \mathrm{dL})$ & 8.1 & 8.4 & 9.0 & 9.3 & ns & ns & ns & ns \\
\hline WBC $\left(\times 10^{9} / \mathrm{L}\right)$ & 8.8 & 66.1 & 39.6 & 44.1 & 0.011 & ns & 0.046 & ns \\
\hline PLT $\left(\times 10^{9} / \mathrm{L}\right)$ & 56.0 & 29.0 & 96.0 & 50.5 & ns & ns & ns & ns \\
\hline PB blasts (\%) & 38.5 & 85.0 & 60.0 & 64.0 & 0.006 & ns & ns & ns \\
\hline BM blasts (\%) & 60.2 & 87.0 & 58.4 & 61.2 & 0.001 & ns & ns & ns \\
\hline
\end{tabular}

Abbreviations: FAB, French-American-British classification; CR, complete remission; FLT3 ITD, fms-like tyrosine kinase3 internal tandem duplication; NN, negative at diagnosis and relapse; PN, positive at diagnosis and negative at relapse; NP, negative at diagnosis and positive at relapse; PP, positive at diagnosis and relapse; ns, not significant; $\mathrm{Hb}$, hemoglobin; WBC, white blood cell; PLT, platelet; PB, peripheral blood; $\mathrm{BM}$, bone marrow; SCT, stem cell transplantation; NPM1, nucleophosmin.

Table 2. Comparison of prognosis between patients with different paired FLT3 ITD mutation status.

\begin{tabular}{|c|c|c|c|c|c|c|c|c|}
\hline \multirow{2}{*}{ Prognostic variables } & \multicolumn{4}{|c|}{ FLT3 ITD mutation status } & \multicolumn{4}{|c|}{$P$} \\
\hline & NN & $\mathrm{PN}$ & NP & PP & NN vs. PN & NN vs. NP & NN vs. PP & PN vs. PP \\
\hline Duration of CR, median (months) & 10.3 & 6.6 & 5.4 & 6.9 & ns & ns & 0.045 & $\mathrm{~ns}$ \\
\hline Overall survival, median (months) & 19.5 & 45.5 & 9.2 & 12.1 & ns & 0.002 & 0.003 & 0.025 \\
\hline Survival after relapse, median (months) & 10.3 & 43.5 & 5.3 & 4.9 & ns & 0.017 & 0.024 & 0.028 \\
\hline
\end{tabular}

Abbreviations: CR, complete remission; FLT3 ITD, fms-like tyrosine kinase3 internal tandem duplication; NN, negative at diagnosis and relapse; $\mathrm{PN}$, positive at diagnosis and negative at relapse; NP, negative at diagnosis and positive at relapse; PP, positive at diagnosis and relapse; ns, not significant.

between groups with different FLT3 ITD mutation status at diagnosis and relapse. A comparison between the NN+NP group, which comprised patients with negative FLT3 ITD mutation status at diagnosis, and the $\mathrm{PN}+\mathrm{PP}$ group, which included patients with positive FLT3 ITD mutation status at diagnosis, showed that the median WBC count at diagnosis was significantly higher in the $\mathrm{PN}+\mathrm{PP}$ group $\left(44.1 \times 10^{9} / \mathrm{L}\right)$ than in the NN+NP group $\left(12.4 \times 10^{9} / \mathrm{L} ; P=0.007\right)$. Moreover, 
Table 3. Comparison of clinical variables and prognosis between groups with different FLT3 ITD mutation status at diagnosis and relapse.

\begin{tabular}{|c|c|c|c|c|c|c|}
\hline \multirow[b]{2}{*}{ Clinical variables } & \multicolumn{4}{|c|}{ FLT3 ITD status (\% with each status) } & \multicolumn{2}{|c|}{$P$} \\
\hline & $\begin{array}{l}\mathrm{NN}+\mathrm{NP} \text { (Negative } \\
\text { at diagnosis) }\end{array}$ & $\begin{array}{l}\mathrm{PN}+\mathrm{PP} \text { (Positive } \\
\text { at diagnosis) }\end{array}$ & $\begin{array}{l}\mathrm{NN}+\mathrm{PN} \text { (Negative } \\
\text { at relapse) }\end{array}$ & $\begin{array}{l}\mathrm{NP}+\mathrm{PP} \text { (Positive } \\
\text { at relapse) }\end{array}$ & $\begin{array}{l}\mathrm{NN}+\mathrm{NP} \text { vs. } \\
\mathrm{PN}+\mathrm{PP}\end{array}$ & $\begin{array}{l}\mathrm{NN}+\mathrm{PN} \text { vs. } \\
\mathrm{NP}+\mathrm{PP}\end{array}$ \\
\hline $\operatorname{Sex}(M: F)$ & $29: 20$ & $13: 7$ & $30: 19$ & $12: 8$ & ns & ns \\
\hline Age, median (range) & $\begin{array}{c}56.5 \\
(5.0-79.0)\end{array}$ & $\begin{array}{c}41.0 \\
(5.0-79.0)\end{array}$ & $\begin{array}{c}53.0 \\
(6.0-79.0)\end{array}$ & $\begin{array}{c}55.0 \\
(5.0-79.0)\end{array}$ & ns & ns \\
\hline \multicolumn{7}{|l|}{ SCT in $1^{\text {st }}$ CR status } \\
\hline No & $36(73.5)$ & $12(66.7)$ & $35(71.4)$ & $13(65.0)$ & ns & ns \\
\hline Yes & $13(26.5)$ & $8(33.3)$ & 14 (28.6) & $7(35.0)$ & & \\
\hline \multicolumn{7}{|l|}{ SCT after $1^{\text {st }}$ relapse } \\
\hline No & $36(73.5)$ & $11(55.0)$ & $39(79.6)$ & $8(33.3)$ & ns & $<0.001$ \\
\hline Yes & $13(26.5)$ & $9(45.0)$ & $10(20.4)$ & $12(66.7)$ & & \\
\hline \multicolumn{7}{|c|}{ Laboratory findings at diagnosis, median } \\
\hline $\mathrm{Hb}(\mathrm{g} / \mathrm{dL})$ & 8.3 & 8.6 & 8.1 & 8.9 & ns & ns \\
\hline WBC $\left(\times 10^{9} / \mathrm{L}\right)$ & 12.4 & 44.1 & 17.1 & 39.5 & 0.007 & ns \\
\hline PLT $\left(\times 10^{9} / \mathrm{L}\right)$ & 58.0 & 41.0 & 51.0 & 69.0 & ns & ns \\
\hline PB blasts (\%) & 47.0 & 68.0 & 55.0 & 60.0 & 0.006 & ns \\
\hline BM blasts (\%) & 61.3 & 87.0 & 64.6 & 64.2 & 0.005 & ns \\
\hline Duration of CR median (months) & 8.6 & 6.9 & 10.0 & 6.8 & ns & 0.034 \\
\hline Overall survival, median (months) & 17.6 & 13.7 & 23.1 & 12.0 & ns & $<0.001$ \\
\hline Survival after relapse, median (months & 6.9 & 6.4 & 11.8 & 5.5 & ns & $<0.001$ \\
\hline
\end{tabular}

Abbreviations: CR, complete remission; Hb, hemoglobin; WBC, white blood cell; PLT, platelet; PB, peripheral blood; BM, bone marrow; SCT, stem cell transplantation; FLT3 ITD, fms-like tyrosine kinase3 internal tandem duplication; NN, negative at diagnosis and relapse; PN, positive at diagnosis and negative at relapse; NP, negative at diagnosis and positive at relapse; PP, positive at diagnosis and relapse; ns, not significant.

A

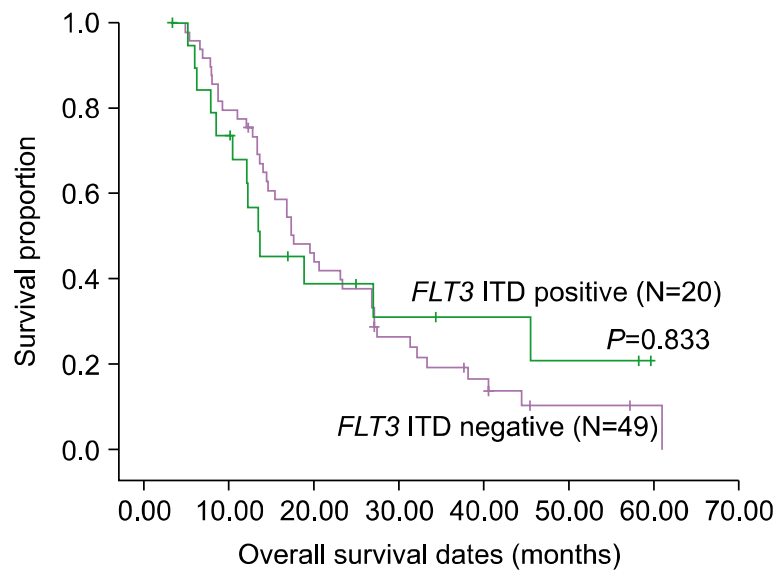

B

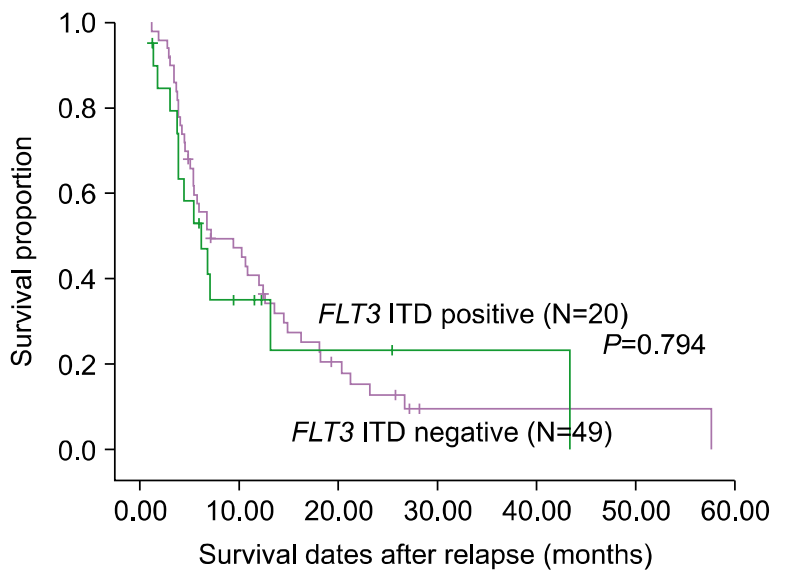

Fig. 1. Comparison of overall survival (A) and survival after relapse (B) between patients positive and negative for FLT3 ITD mutation at diagnosis.

the $\mathrm{PN}+\mathrm{PP}$ group showed significantly higher blast percentage in $\mathrm{PB}(68.0 \%$ vs. $47.0 \% ; P=0.006)$ and in $\mathrm{BM}(87.0 \%$ vs. $61.3 \%, P=0.005)$ than the $\mathrm{NN}+\mathrm{NP}$ group did. However, there was no difference in the duration of $C R$, there was no difference in OS and survival after relapse between these groups (Fig. 1). Another comparison was performed between the $\mathrm{NN}+\mathrm{PN}$ group, which included patients with negative FLT3 ITD mutation status at relapse, and the NP+PP group, which included patients with positive FLT3 ITD mutation status at relapse, revealed that the median duration of $\mathrm{CR}$ was significantly shorter in the $\mathrm{NP}+\mathrm{PP}$ group (6.8 months) than in the $\mathrm{NN}+\mathrm{PN}$ group (10.0 months; $P=0.034)$. In partic- ular, the median OS was significantly shorter in the NP+PP group (12.0 months) than in the $\mathrm{NN}+\mathrm{PN}$ group (23.1 months; $P<0.001$ ), as was survival after relapse (5.5 vs. 11.8 months; $P<0.001)$, despite the fact that SCT was performed significantly more frequently in the $\mathrm{NP}+\mathrm{PP}$ group than in the $\mathrm{NN}+\mathrm{PN}$ group after the first relapse $(66.7 \%$ vs. $20.4 \% ; P$ $<0.001$ ) (Fig. 2).

4. Comparison of prognosis in patients without FLT3 ITD mutation stratified by NPM1 genotype status at diagnosis and relapse

Patients with NPM1 mutation at diagnosis showed a trend 

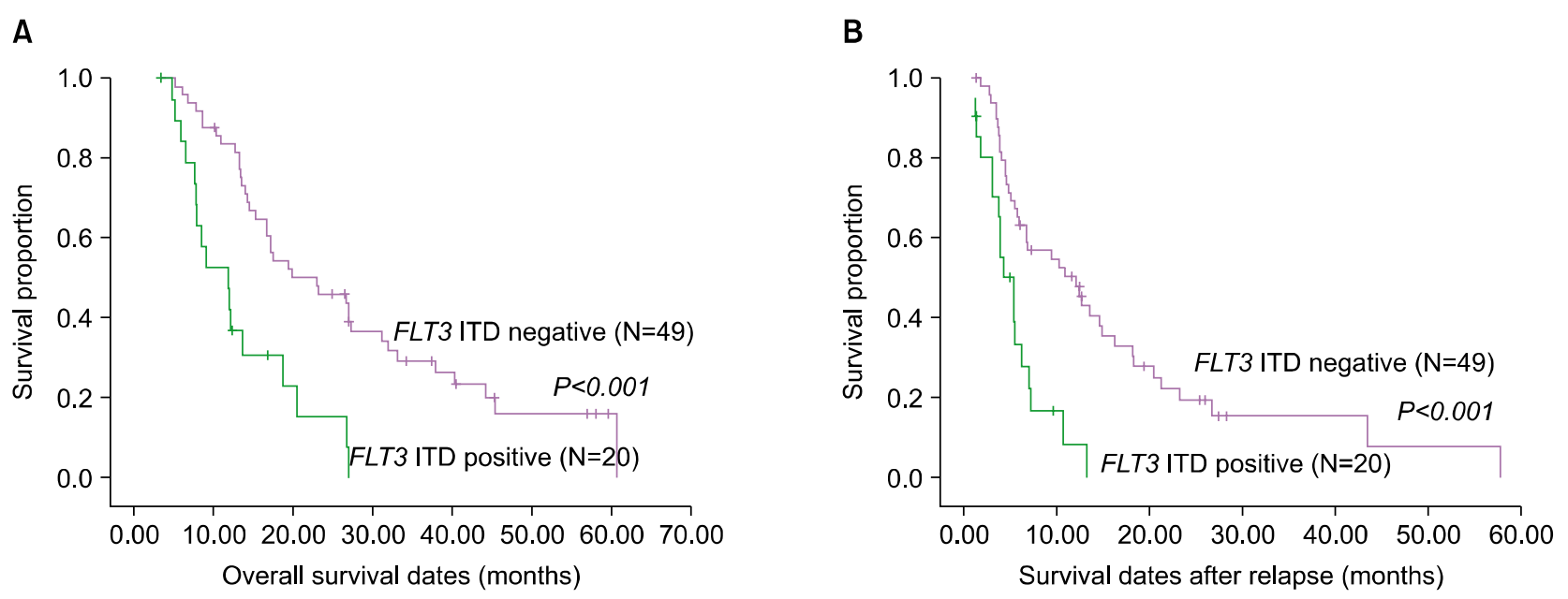

Fig. 2. Comparison of overall survival (A) and survival after relapse (B) between patients positive and negative for FLT3 ITD mutation at relapse.

Table 4. Multivariate analysis of OS and survival after relapse with respect to FLT3 ITD mutation status at diagnosis and relapse.

\begin{tabular}{|c|c|c|c|c|}
\hline \multirow{2}{*}{ Clinical variables } & \multicolumn{2}{|c|}{ Overall survival } & \multicolumn{2}{|c|}{ Survival after relapse } \\
\hline & $\mathrm{HR}(95 \% \mathrm{Cl})$ & $P$ & $\mathrm{HR}(95 \% \mathrm{Cl})$ & $P$ \\
\hline $\begin{array}{l}\text { FLT3 ITD mutation at relapse } \\
\text { (compared with negative at relapse) }\end{array}$ & $2.486(1.203-5.138)$ & $0.014^{\text {a) }}$ & $2.042(1.062-4.045)$ & $0.039^{a)}$ \\
\hline $\begin{array}{l}\text { FLT3 ITD mutation at diagnosis } \\
\text { (compared with negative at diagnosis) }\end{array}$ & $1.084(0.528-2.144)$ & $\mathrm{ns}^{\mathrm{b})}$ & $1.147(0.534-2.447)$ & $\mathrm{ns}^{\mathrm{b})}$ \\
\hline
\end{tabular}

towards a better outcome, such as longer OS (24.6 vs. 17.3 months; $P=0.173)$ and duration of CR (10.0 vs. 7.5 months; $P=0.558)$ than those without NPM1 mutation at diagnosis. However, these differences were not statistically significant. Patients with NPM1 mutation at relapse also showed no differences in OS (15.4 vs. 23.1 months; $P=0.609)$ and duration of CR (8.6 vs. 10.3 months; $P=0.657)$ compared with those without $N P M 1$ mutation at relapse.

5. Comparison of prognosis in patients with FLT3 ITD mutation at diagnosis or relapse according to the amount of FLT3 ITD mutants

The high/low cut-off ratio was set at 0.66 , as described in a previous study [23]. Six patients with a high mutant:wild ratio $(\geq 0.66)$ at diagnosis showed trends towards shorter OS (9.3 vs. 22.5 months; $P=0.370$ ) and duration of CR (4.1 vs. 6.6 months; $P=0.682$ ) than 14 patients with a low ratio $(<0.66)$ at diagnosis, suggesting possible poor prognosis, but the results were not statistically significant. Eight patients with high mutant:wild ratios $(\geq 0.66)$ at relapse also showed similar OS (9.2 vs. 10.1 months; $P=0.734$ ) and duration of CR (4.1 vs. 5.4 months; $P=0.701$ ) as shown by 12 patients with a low ratio $(<0.66)$ at relapse.
6. Multivariate analysis of $O S$ and survival after relapse related to FLT3 ITD mutation status at diagnosis and relapse Table 4 shows the results of multivariate analysis of OS and survival after relapse related to FLT3 ITD mutation status at diagnosis and relapse. FLT3 ITD mutation at relapse, irrespective of the initial mutation status, showed a statistically significant association with a poor prognosis on OS (HR, 2.486; $P=0.014)$ and survival after relapse (HR, 2.042; $P=$ 0.039). FLT3 ITD mutation at diagnosis did not show statistically significant prognostic impact on OS or survival after relapse.

\section{DISCUSSION}

In this study, the FLT3 ITD mutation was present in 20 (29.0\%) patients each at diagnosis and relapse, similar to the findings of a recent study [22]. Between diagnosis and relapse, the FLT3 ITD mutation status changed in 18 patients (26.1\%). This figure is quite high relative to the recently reported value of $17.5 \%$, and reflects the heterogeneity of FLT3 ITD mutation populations [24].

Patients with persistent FLT3 ITD mutation showed higher initial WBC counts than those with persistent wild-type 
status. Patients with loss of the FLT3 ITD mutation at relapse showed higher initial WBC count, blast percentage in $\mathrm{PB}$ and $\mathrm{BM}$ than those with persistent wild-type status, consistent with the previous findings [11-16]. Patients who acquired the FLT3 ITD mutation at relapse showed significantly shorter OS and survival after relapse than those with persistent wild-type status, and similar differences were observed between patients who acquired the FLT3 ITD mutation at relapse and patients with persistent FLT3 ITD mutation. Additionally, patients with persistent FLT3 ITD mutation showed a significantly shorter duration of CR than those with persistent wild-type status. These findings were most pronounced in patients with FLT3 ITD mutation at relapse, compared to those with mutation-negative status at relapse, despite SCT being significantly more frequently performed after the first relapse in patients with FLT3 ITD mutation. Collectively, the results suggest that FLT3 ITD mutation at relapse is a poor prognostic indicator, and are consistent with recent data that reported a shorter time-to-relapse in patients with FLT3 ITD mutation at relapse compared to those without the mutation [24]. On the other hand, FLT3 ITD mutation status at diagnosis influenced WBC count and blast percentage in $\mathrm{PB}$ and $\mathrm{BM}$ at diagnosis, but prognostic differences according to mutation status at diagnosis were not statistically significant. These differences between the prognostic potential of mutation status at diagnosis and relapse may indicate that the prognostic impact of FLT3 ITD mutation status at relapse is more pronounced than the mutation status at diagnosis in normal-karyotype AML patients with relapse.

Multivariate analysis revealed the FLT3 ITD mutation at relapse to be an independent indicator of poor prognosis for both OS and survival after relapse. Otherwise, the presence of FLT3 ITD mutation at diagnosis did not show any prognostic effect. These results support our speculation that the impact of FLT3 ITD mutation status at relapse is of greater prognostic value than the mutation status at diagnosis in normal-karyotype AML patients with relapse. Thus far, FLT3 ITD mutation status at diagnosis has been firmly established as a prognostic indicator for normal-karyotype AML $[25,26]$. Moreover, the ratio of mutant:wild-type cells has been suggested to be a possible prognostic indicator by a previous study, which showed significantly lower 5-year relapse-free survival rates $(57 \%$ vs. $79 \%, P=0.048)$ in patients with a high mutant:wild-type ratio $(\geq 0.66)$ than in those with a low ratio. However, in our study, not only did the FLT3 ITD mutation status at diagnosis not predict outcome but there was also no evidence of a prognostic impact of the relative amount of the mutant cells. One possible explanation for the discordant results is the different nature of the patient group included in the analysis. Previous studies have focused on FLT3 ITD mutation status at initial diagnosis; therefore, all patients could be included into analysis regardless of their status at relapse. However, in our study, only patients who had undergone a relapse could be enrolled. Because the clinical outcome between relapsed and nonrelapsed patients is quite different, this heterogeneity might influence the clinical outcome.

Our study had some limitations. First, several molecular aberrations which have potential prognostic impacts in normal karyotype AML, such as CCAAT-enhancer binding protein- $\alpha(C E B P A)$ and Wilm's tumor 1 (WT1), were not analyzed. These factors may have influenced our results. Second, the number of patients was relatively small, which may lead to false positives or negatives and thus would have affected our conclusions. Third, although we found that a prognostic impact of $N P M 1$ mutation was not evident at either diagnosis or relapse in patients in the absence of FLT3 ITD mutation, this analysis suffered from low statistical power due to the limited number of patients. For this reason, we could not perform more categorized analysis based on the different mutation status combination of FLT3 ITD and NPM1, which has been widely used in recent studies. Therefore, further studies with larger patient populations will be required for more accurate analysis.

In conclusion, patients with acquired or persistent FLT3 ITD mutation at relapse showed significantly shorter OS and survival after relapse than those with persistent wildtype status. These statistical differences were most pronounced in patients with FLT3 ITD mutation at relapse, when compared to those with mutation-negative status at this time point. However, the mutation status at diagnosis did not affect the outcome. Collectively, these results suggest that the impact of FLT3 ITD mutation status at relapse is expected to be of greater prognostic value than the mutation status at diagnosis in normal karyotype-AML patients with relapse.

\section{REFERENCES}

1. Gilliland DG, Griffin JD. The roles of FLT3 in hematopoiesis and leukemia. Blood 2002;100:1532-42.

2. Brasel K, Escobar S, Anderberg R, de Vries P, Gruss HJ, Lyman SD. Expression of the flt 3 receptor and its ligand on hematopoietic cells. Leukemia 1995;9:1212-8.

3. Drexler HG. Expression of FLT3 receptor and response to FLT3 ligand by leukemic cells. Leukemia 1996;10:588-99.

4. Turner AM, Lin NL, Issarachai S, Lyman SD, Broudy VC. FLT3 receptor expression on the surface of normal and malignant human hematopoietic cells. Blood 1996;88:3383-90.

5. Birg F, Courcoul M, Rosnet O, et al. Expression of the FMS/ KIT-like gene FLT3 in human acute leukemias of the myeloid and lymphoid lineages. Blood 1992;80:2584-93.

6. Carow CE, Levenstein M, Kaufmann SH, et al. Expression of the hematopoietic growth factor receptor FLT3 (STK-1/Flk2) in human leukemias. Blood 1996;87:1089-96.

7. Stacchini A, Fubini L, Severino A, Sanavio F, Aglietta M, Piacibello W. Expression of type III receptor tyrosine kinases FLT3 and KIT and responses to their ligands by acute myeloid leukemia blasts. Leukemia 1996;10:1584-91.

8. Levis M, Small D. FLT3: ITDoes matter in leukemia. Leukemia 2003;17:1738-52.

9. Hayakawa F, Towatari M, Kiyoi H, et al. Tandem-duplicated Flt3 
constitutively activates STAT5 and MAP kinase and introduces autonomous cell growth in IL-3-dependent cell lines. Oncogene 2000;19:624-31.

10. Yamamoto Y, Kiyoi H, Nakano Y, et al. Activating mutation of D835 within the activation loop of FLT3 in human hematologic malignancies. Blood 2001;97:2434-9.

11. Abu-Duhier FM, Goodeve AC, Wilson GA, et al. FLT3 internal tandem duplication mutations in adult acute myeloid leukaemia define a high-risk group. Br J Haematol 2000;111:190-5.

12. Fröhling S, Schlenk RF, Breitruck J, et al. Prognostic significance of activating FLT3 mutations in younger adults (16 to 60 years) with acute myeloid leukemia and normal cytogenetics: a study of the AML Study Group Ulm. Blood 2002;100:4372-80.

13. Kottaridis PD, Gale RE, Frew ME, et al. The presence of a FLT3 internal tandem duplication in patients with acute myeloid leukemia (AML) adds important prognostic information to cytogenetic risk group and response to the first cycle of chemotherapy: analysis of 854 patients from the United Kingdom Medical Research Council AML 10 and 12 trials. Blood 2001;98:1752-9.

14. Kiyoi H, Naoe T, Nakano Y, et al. Prognostic implication of FLT3 and N-RAS gene mutations in acute myeloid leukemia. Blood 1999;93:3074-80.

15. Kottaridis PD, Gale RE, Linch DC. Flt3 mutations and leukaemia. Br J Haematol 2003;122:523-38.

16. Thiede C, Steudel C, Mohr B, et al. Analysis of FLT3-activating mutations in 979 patients with acute myelogenous leukemia: association with FAB subtypes and identification of subgroups with poor prognosis. Blood 2002;99:4326-35.

17. Mead AJ, Linch DC, Hills RK, Wheatley K, Burnett AK, Gale RE. FLT3 tyrosine kinase domain mutations are biologically distinct from and have a significantly more favorable prognosis than FLT3 internal tandem duplications in patients with acute myeloid leukemia. Blood 2007;110:1262-70.

18. Whitman SP, Ruppert AS, Radmacher MD, et al. FLT3 D835/I836 mutations are associated with poor disease-free survival and a distinct gene-expression signature among younger adults with de novo cytogenetically normal acute myeloid leukemia lacking FLT3 internal tandem duplications. Blood 2008;111:1552-9.

19. Mead AJ, Gale RE, Hills RK, et al. Conflicting data on the prognostic significance of FLT3/TKD mutations in acute myeloid leukemia might be related to the incidence of biallelic disease. Blood 2008;112:444-5.

20. Rau R, Brown P. Nucleophosmin (NPM1) mutations in adult and childhood acute myeloid leukaemia: towards definition of a new leukaemia entity. Hematol Oncol 2009;27:171-81.

21. Ferrara F, Palmieri S, Leoni F. Clinically useful prognostic factors in acute myeloid leukemia. Crit Rev Oncol Hematol 2008;66: 181-93.

22. Huang Q, Chen W, Gaal KK, Slovak ML, Stein A, Weiss LM. A rapid, one step assay for simultaneous detection of FLT3/ITD and NPM1 mutations in AML with normal cytogenetics. Br J Haematol 2008;142:489-92.

23. Chillón MC, Santamaría C, García-Sanz R, et al. Long FLT3 internal tandem duplications and reduced PML-RARalpha expression at diagnosis characterize a high-risk subgroup of acute promyelocytic leukemia patients. Haematologica 2010;95:74551.

24. Cloos J, Goemans BF, Hess CJ, et al. Stability and prognostic influence of FLT3 mutations in paired initial and relapsed AML samples. Leukemia 2006;20:1217-20.

25. Kim YK, Kim HN, Lee SR, et al. Prognostic significance of nucleophosmin mutations and FLT3 internal tandem duplication in adult patients with cytogenetically normal acute myeloid leukemia. Korean J Hematol 2010;45:36-45.

26. Chang SH, Lee NY, Kim DH, Sohn SK, Suh JS. FLT3 Gene Mutations as a Prognostic Factor for Acute Myeloid Leukemia. Korean J Lab Med 2006;26:233-40. 